\title{
MICROTURBINA PELTON, UNA SOLUCION REAL DE ENERGIA PARA ZONAS NO INTERCONECTADAS (ZNI)
}

\section{MICROTURBINA PELTON, A REAL SOLUTION OF ENERGY FOR ZONES NOT INTERCONNECTED (ZNI)}

\author{
Esp. Alfredo Bohórquez Niño \\ Universidad Francisco de paula Santander Ocaña \\ Facultad de Ingenierías, Grupo GITYD \\ Vía acolsure Sede Algodonal, Ocaña, Colombia \\ (57) 5690088 ext:221 \\ abohorquezni@ufpso.edu.co
}

\begin{abstract}
RESUMEN
"La energía no se crea ni se destruye, solo se transforma" lo manifestó por primera vez el científico Antoine Lavoisier en el año 1785. Se expone a continuación el desarrollo de una solución energética aplicada en las zonas no interconectadas, concretamente la micro turbina tipo Pelton, que aprovecha pequeñas caídas de agua y transforma esa energía cinética en fuerza motriz suficiente para hacer girar un alternador y hacer funcionar los electrodomésticos requeridos en una o varias viviendas relativamente cercanas.

El presente trabajo muestra el desarrollo desde el diseño, la construcción y puesta en funcionamiento de este sistema compacto con todos los elementos requeridos para poderlo trasladar de manera práctica, se describe la aplicación real en varias regiones no interconectadas de nuestro país.
\end{abstract}

Palabras clave: micro turbina pelton, energía, agua. Sostenible

\begin{abstract}
:
"Energy is not created or destroyed, it is only transformed" was first stated by the scientist Antoine Lavoisier in 1785. The development of an energy solution applied in noninterconnected areas, specifically the micro turbine type, is presented below. Pelton, which takes advantage of small drops of water and transforms that kinetic energy into enough motive power to turn an alternator and operate the required appliances in one or several relatively close homes.

The present work shows the development from the design, construction and start-up of this compact system with all the elements required to be able to move it in a practical way, the actual application is described in several non-interconnected regions of our country.
\end{abstract}

Keywords: Pelton micro turbine, energy, water. sustainable. 


\section{INTRODUCCIÓN}

El sector rural, especialmente el que se encuentra en las zonas no interconectadas, carece de uno de los principales servicios básicos para poder tener una mejor calidad de vida, la energía eléctrica.

Los campesinos tienen pocas oportunidades de manejar sus productos perecederos debido a la falta de cadenas de frio que impidan el deterioro de los productos por los largos espacios y tiempos de recorrido para recolectar los productos. Lo ideal sería que en las veredas se pudiera garantizar un sistema de enfriamiento para que los productos lleguen en buen estado a las cabeceras municipales (Monroy Gonzales M. F. \& López Martínez G. A., 2012).

La hidrogeneración de energía en pequeña escala en potencias por debajo de los $500 \mathrm{Kw}$ constituye una alternativa a la solución del problema de suministro de energía en regiones aisladas, especialmente en los países en vías de desarrollo, constituyéndose así en una de las bases principales para la electrificación rural.(sanchez \& Ramirez Gastón, 1995)

En Colombia las pequeñas centrales hidroeléctricas PCHs se definen bajo los intervalos establecidos por la Comisión de Regulación de Energía y Gas CREG así: en el rango de 0 a 100 kilovatios Micro central, de 100 a 1000 kilovatios Mini central y de 1000 a 10.000 kilovatios Pequeña central: (Sierra, Sierra, \& Guerrero, 2011). La Energia electrica es un recurso limitado que debe utilizarce eficientemente, con reducido impacto ambiental y al menor costo. (Arévalo,Cabellos \& García, 2017)

En el mismo sector rural se encuentra gran parte de la solución al problema planteado, se hace necesario desarrollar una

Estructura portátil y de fácil instalación para trasladar la alternativa de solución al lugar donde se requiere.

El desarrollo de la Robótica agrícola es posible, dado que la carga de las baterías necesaria para el funcionamiento de dichos equipos, se puede obtener en el horario nocturno cuando el campesino no requiere de la energía de la micro central en su vivienda. (Torres, C., Archila,J.,Tronco, M., Becker, M., Viera, A., Tiberti, A., 2012)

El registro a distancia de fenómenos climatológicos, es posible mediante la utilización de equipos tecnológicos como el Fluviógrafo electrónico con transmisión de datos inalámbrica que requiere energía para su funcionamiento ininterrumpido. La cual pude ser suministrada por la micro central hidroeléctrica planteada. (Sandoval, Tobar, Mosquera \& Gonzales.,2010)

\section{FASE DE DISEÑO}

Las propiedades de los elementos mecánicos son un factor importante a considerar en el diseño de un equipo en aspectos como operación y seguridad del operario. (Espinel,Yaruro \& Gerrero,2017)

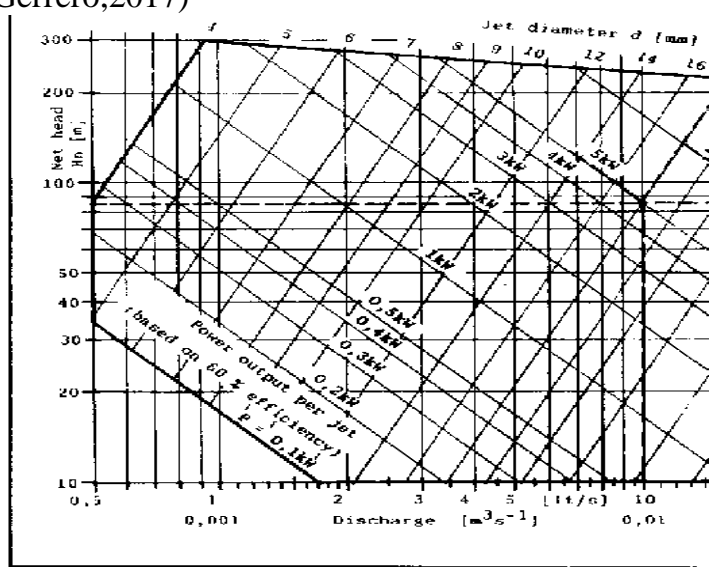

Figura 1. Cuadro de potencias (Altura vs Caudal)

Fuente: Micro Pelton turbines Niederuzwil, September 1991

El diseño de la micro turbina contempla la aplicación de tres rodetes monolíticos de diferentes diámetros para cubrir respectivamente, gran parte de las diferentes características topográficas y de caudal que presenta nuestra geografía andina.

Concretamente el sistema capta mediante una bocatoma un determinado caudal de agua en la parte elevada de un terreno y desciende por manguera hasta la parte más baja, donde se ubica el equipo electromecánico, es decir la Micro turbina acoplada con el alternador. Dicho caudal retorna al cauce inicial, sin contaminarse. A este sistema se le denomina Micro central Hidroeléctrica.

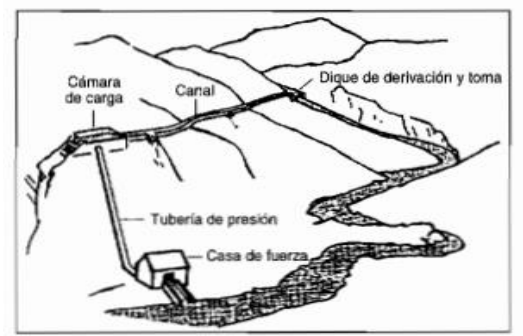

Figura 2. Esquema de una micro central hidroeléctrica. 
Fuente: ITDG Soluciones prácticas.

El rodete Pelton se aplica en lugares donde se cuenta con gran altura y muy poco caudal y se aplica la siguiente ecuación para el cálculo de La potencia en kilovatios a obtener, Según el Manual de Mini y Micro centrales Hidráulicas de la ITDG del Perú 1995.

$$
P_{\text {neta (estimada) }}=5 \times Q \times h_{\text {disp }} \mathrm{kW}
$$

$\mathrm{P}=$ Potencia neta estimada

$5=$ Factor que involucra las pérdidas del sistema.

$\mathrm{Q}=$ Caudal .

$\mathrm{h}=$ Altura Disponible.

El caudal disponible define el diámetro del chorro de salida, a partir del cual se diseñan las demás partes de la micro turbina.

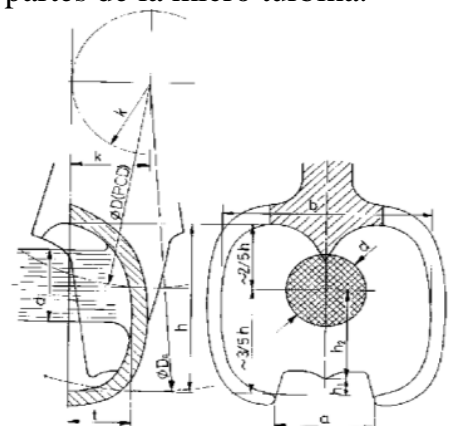

Figura 3. Esquema de cuchara pelton

Fuente: Micro Pelton turbines Niederuzwil, September 1991

Para la fabricación del modelo y el comportamiento bajo cargas, de la carcasa de la turbina se utilizó el software solidwork.

La aplicación de la cultura digital con tecnicas CAD-CAM que permiten obtener la geometría idónea en la obtención del modelo de alta calidad a reproducir.(Avila, Florez \& Gualdron, 2012)

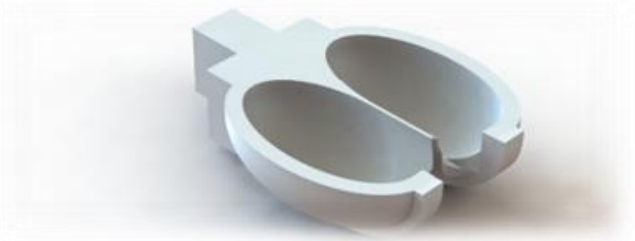

Figura 4. Diseño de cuchara Pelton Fuente: Elaboración propia.

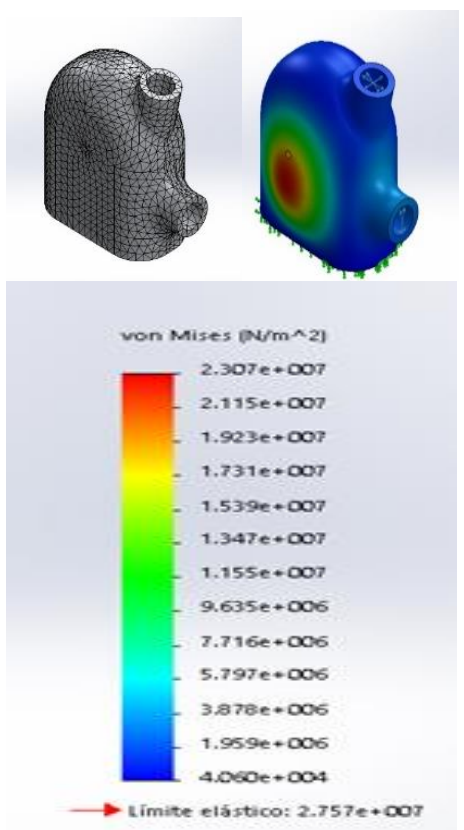

Figura 5. Análisis de la carcasa Fuente: Elaboración propia.

\section{FASE DE CONSTRUCCIÓN}

El Bronce es material seleccionado para la construcción del rodete (fig. 6) dada su alta resistencia a la fricción y su relativo bajo punto de fusión, teniendo en cuenta que no se requiere una gran infraestructura para su procesamiento.

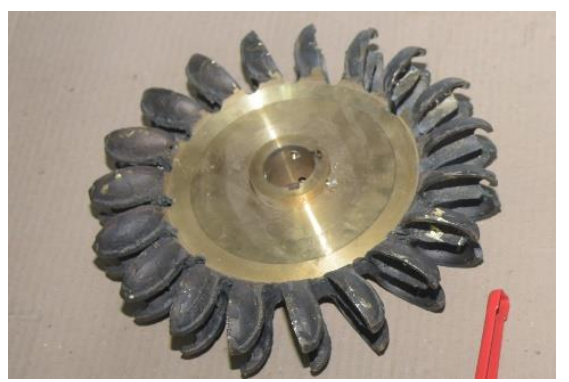

Figura 6. Rodete Pelton en bronce.

Fuente: Elaboración propia

La construcción de la carcasa se realiza por el método de moldeo en arena y el material utilizado es aluminio (fig. 7) dada su alta resistencia a la corrosión para garantizar su estabilidad dimensional y estructural con el paso del tiempo. 


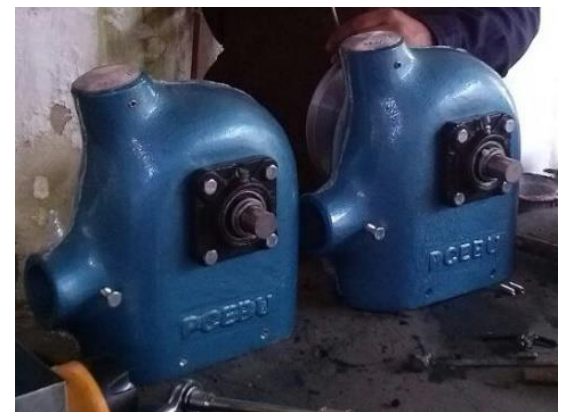

Figura 7. Ensamble de dos carcazas en aluminio. Fuente: Elaboración propia.

El conjunto electromecánico compacto permite ser trasladado al lugar de instalación de manera muy práctica y segura, ya que cuenta con una estructura de protección que rodea tanto al generador como a la turbina. (Fig. 8)

Existen pérdidas técnicas atribuidas a la energía consumida por los dispositivos del sistema que no es aprovechable, por ejemplo disipación en líneas eléctricas y transmisión de potencia mecánica por correa. (Núñez, Hincapié \& Gallego, 2012)

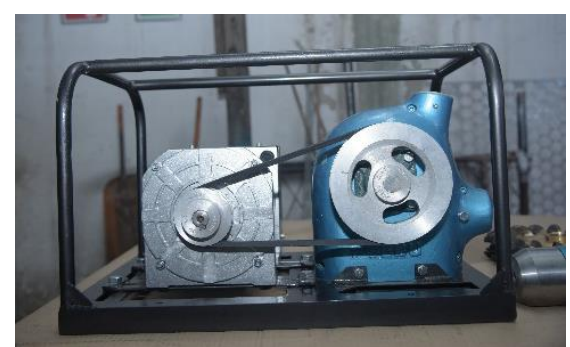

Figura 8. Micro turbina Pelton compacta Fuente: Elaboración propia.

\section{FASE DE MONTAJE.}

Este modelo de micro turbina ha sido instalado y puesto en funcionamiento en diferentes regiones pertenecientes a las zonas no interconectadas de nuestro país, como lo son el municipio de San Vicente del Caguan, (Figura 9), Puerto rico, El Doncello, ubicados en el departamento del Caquetá, el cual cuenta con un amplio territorio montañoso y con abundantes fuentes hídricas que lo hacen idóneo para la aplicación de esta solución de energía alternativa y sustentable.
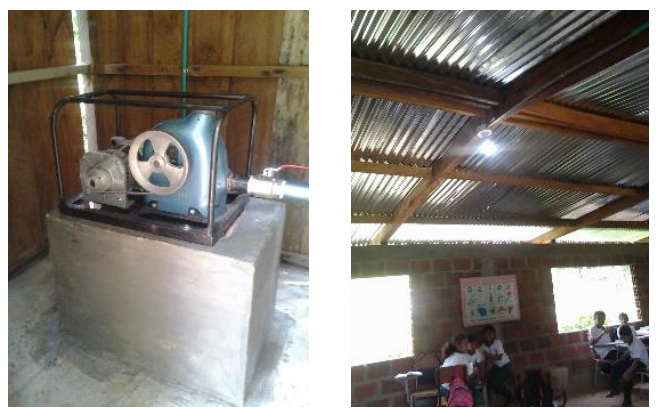

Figura 9. Montaje vereda el Cafeto (San Vicente del Caguan)

Otro departamento donde ha sido posible implementar la micro turbina, ofreciendo energía suficiente para varias viviendas con un solo montaje, es la región del Sarare en el Norte de Santander, específicamente en la reserva natural del Tama, en límites con Venezuela (Figura 10)

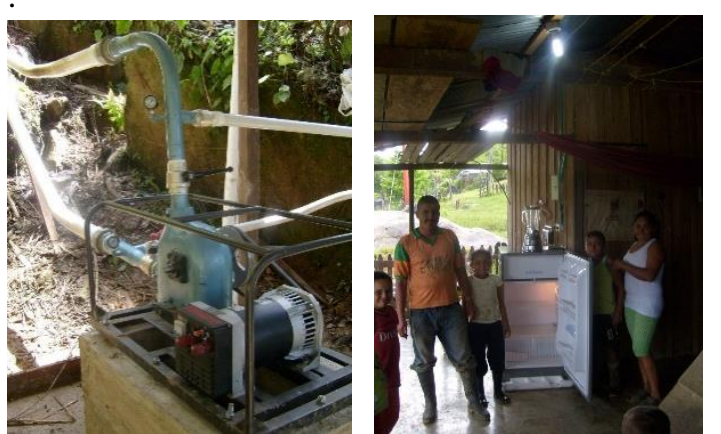

Figura 10. Montaje vereda Santa marta II, municipio de Toledo (Norte de Santander)

De igual forma ha sido aplicada en varias viviendas rurales del área del Catatumbo pertenecientes a los municipios de Convención, Teorama, El tarra, el Zulia, (Figura 11) entre otros.
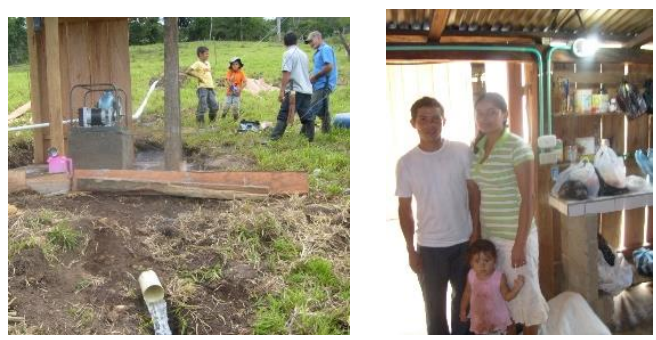

Figura 11. Montaje vereda Jacaranda, municipio de El Zulia (Norte de Santander)

\section{DISCUSIÓN.}

Es un hecho, que la tecnología requerida para el diseño y la fabricación de soluciones alternativas a la problemática energética de las zonas no 
interconectadas ya tiene una aplicación concreta y demostrable. Sin embargo el afán por solucionar problemas de regulación y perfeccionamiento de la calidad de energía eléctrica a suministrar, retarda la aplicación inmediata del sistema a gran escala.

Dicho perfeccionamiento se bebe implementar sobre la marcha, sin dilatar la aplicación en los lugares donde se está requiriendo con prontitud.

\section{CONCLUSIONES.}

- La selección de los materiales tanto del bronce para el rodete, como del aluminio para la carcasa es idónea, considerando que se han sometido a prueba durante 10 años en funcionamiento ininterrumpido $\mathrm{y}$ han cumplido a satisfacción con las expectativas.

- La calidad de la energía eléctrica obtenida, si bien no está lo suficientemente regulada como se quisiera, se encuentra de acuerdo al diseño en un rango de seguridad para no perjudicar el funcionamiento de los electrodomésticos conectados al circuito.

No obstante existen importantes desarrollos en esta área, tales como los convertidores DC-AC usando factor de distorsión armónica total (THD). (Araque, Dias \& Gualdron, 2012).

- La tendencia a mantener un control del costo del equipo en un nivel accesible al usuario hace que no se le implementen equipos de regulación que mejorarían la calidad de la energía suministrada.

\section{REFERENCIAS:}

Araque,J., Diaz, J.,\& Gualdron, O., (2012). Optimización del THD en un convertidor multinivel monofásico usando algoritmos genéticos. Revista Colombiana de Tecnologías de Avanzada ISSN: 1692-7257 1 (21). Pág. 60 $-66$.

Arévalo,J., Cabellos, M., \& García, J (2017).

Consumo de energía eléctrica en diferentes viviendas en la ciudad de Ocaña, Norte de Santander. Revista Colombiana de Tecnologías de Avanzada ISSN: 1692-7257 1 (29). Pág. 42 $-46$.

Avila,A., Florez, E., \& Gualdron, O., (2012) Diseño e implementación de un curso interactivo multimedia para el aprendizaje de los procesos CAM en un centro de mecanizado leadwell v30. Revista Colombiana de Tecnologías de Avanzada ISSN: 1692-7257 2 (20). Pág. $50-56$.

Espinel,E.,Yaruro,J. \& Guerrero,G (2016). Prototipo de máquina para fabricar ensilaje utilizando un tornillo sin fin para la compresión del producto en bolsa plástica. Revista Colombiana de Tecnologías de Avanzada ISSN: 1692-7257 1 (29). Pág. 58 - 63.

ITDG Soluciones Prácticas (n.d). Ficha Técnica N²5 Turbinas Pelton. Página web de la organización Soluciones Prácticas ITDG, LimaPerú.

Monroy Gonzales, M., \& López Martínez, G. A. (23 de OCTUBRE de 2012). VII Congreso Bolivariano de Ingeniería Mecánica. Pequeñas centrales hidroeléctricas (pch). Alternativa de energía sustentable en latinoamerica y el caribe, 4. cusco, peru.

Nuñez,P., Hincapie,R. \& Gallego,R. (2012).

Metodología para reubicación de transformadores de distribución considerando el sistema de protección. Revista Colombiana de Tecnologías de Avanzada ISSN: 1692-7257 2 (20). Pág. 28 -35 .

Sanchez, T., \& Ramirez Gastón, J. (1995). Manual de Microcentrales Hidroeléctricas. LIMA: Banco Interamericano de Desarrollo.

Sandoval,G., Tobar,J., Mosquera,V. \& Gonzales,L.,(2010) Pluviógrafo electrónico con transmisión de datos inalámbrica. Revista Colombiana de Tecnologías de Avanzada ISSN: 1692-7257. Pág. $67-73$.

Sierra, F., Sierra, A., \& Guerrero, C. (2011). Pequeñas y microcentrales hidroelectricas: Alternativa real de generación eléctrica. Informador técnico Colombia, 73-85.

Torres, C., Archila ,J. Tronco, M., Becker, M., Viera, A., Tiberti, A., (2012) Estudio cinemático de una plataforma robótica para agricultura. Revista Colombiana de Tecnologías de Avanzada ISSN: 1692-7257 2 (22). Pág. $131-137$. 